\title{
Calculation of steady three-dimensional deep-water waves
}

\author{
By DANIEL I. MEIRON, PHILIP G. SAFFMAN \\ AND HENRY C. YUEN† \\ Applied Mathematics, California Institute of Technology, Pasadena, California 91125
}

(Received 22 January 1981 and in revised form 25 June 1982)

\begin{abstract}
Steady three-dimensional symmetric wave patterns for finite-amplitude gravity waves on deep water are calculated from the full unapproximated water-wave equations as well as from an approximate equation due to Zakharov. These solutions are obtained as bifurcations from plane Stokes waves. The results are in good agreement with the experimental observations of $\mathrm{Su}$.
\end{abstract}

\section{Introduction}

Following the discovery by Chen \& Saffman (1980) of non-uniform two-dimensional steady gravity waves of finite amplitude on deep water, Saffman \& Yuen (1980) (hereafter referred to as SY) demonstrated the existence of steady, fully threedimensional waves of finite amplitude using an approximate equation valid for weakly nonlinear waves. These solutions were obtained as bifurcations from plane (Stokes) waves at wave amplitudes that depend on the longitudinal and transverse periods of the wave pattern. It was pointed out in SY that the bifurcation is degenerate since there are two families of three-dimensional waves, one symmetric about the direction of propagation, and the other skewed. Saffman \& Yuen were unaware of any observations of such three-dimensional waves when the work was done, and they presented only details of the skewed patterns.

Experimental observations by $\mathrm{Su}(1982)$ in both an open channel and a wave tank have demonstrated the existence of steady three-dimensional symmetric wave patterns of a form similar to those predicted by the bifurcation analysis. Here we present results for symmetric wave patterns using the exact equations for inviscid deep-water waves, and compare them with the results obtained with the approximate (Zakharov) equation used by SY as well as the experimental observations of Su (1982).

\section{Formulation}

We consider irrotational waves of permanent form in which the free surface has the form

$$
\eta(x, y, t)=\sum_{l=1}^{\infty} \sum_{m=-\infty}^{\infty} \sum_{n=0}^{\infty} A_{l, m, n}(p, q) \cos [(l+m p(x-c t)] \cos n q y
$$

where $p$ and $q$ are given real numbers. This describes a steady, symmetrical wave pattern propagating in the $x$-direction with constant speed $c$. The free-surface pattern is periodic in the transverse $y$-direction with wavelength $2 \pi / q$. It is in general not exactly periodic in the $x$-direction, unless $p$ is a rational number. Note also that in this case the representation (1) is not unique, but is easily made so, for example by

$\uparrow$ Present address: Fluid Mechanics Department. TRW Space and Technology Group, One Npace Park, Redondo Beach, California 90278. 
imposing the convention that $A_{l, m, n}=0$ if $m p$ is an integer. The special case when $A_{l, m, n}=0$ for $m \neq 0, n \neq 0$ describes a plane wave of wavelength $2 \pi$ with Fourier components $A_{l, \mathbf{0}, 0}$.

These waves satisfy the nonlinear system of equations $(g=1)$

$$
\begin{aligned}
-c \eta_{x}+\phi_{x} \eta_{x}+\phi_{y} \eta_{y} & =\phi_{z}, \quad z=\eta(x, y, t), \\
-c \phi_{x}+\frac{1}{2}\left(\phi_{x}^{2}+\phi_{y}^{2}+\phi_{z}^{2}\right)+\eta & =K, \quad z=\eta(x, y, t), \\
\phi_{x x}+\phi_{y y}+\phi_{z z} & =0, \quad-\infty<z<\eta(x, y, t) .
\end{aligned}
$$

In order to satisfy (4) and have the appropriate symmetry, the potential $\phi$ has the form

$$
\begin{aligned}
& \phi(x, y, z, t)=\sum_{l=1}^{\infty} \sum_{m=-\infty}^{\infty} \sum_{n=0}^{\infty} B_{l, m, n}(p, q) \exp \left(\omega_{l, m, n} z\right) \times \sin [(l+m p)(x-c t)] \cos n q y, \\
& \text { where } \\
& \omega_{l, m, n}=\left[(l+m p)^{2}+n^{2} q^{2}\right]^{\frac{1}{2}} .
\end{aligned}
$$

The mean level, or equivalently the Bernoulli constant $K$, is arbitrary and each may be fixed by specifying the other. For definiteness, we shall regard the mean level as given and the Bernoulli constant as unknown in our calculations. Substitution of the expressions (1). (5) and (6) into the boundary conditions (2) and (3) generates an infinite set of nonlinear equations for the Fourier coefficients $A_{l, m, n}, B_{l, m, n}$, the wave speed $c$ and the Bernoulli constant $K$.

The experience in calculating two-dimensional solutions by Chen \& Saffman (1980) and Saffman (1980) is that in this case the system (2)-(4) is closed and gives isolated (but not necessarily unique) solutions if one additional quantity such as the waveheight (or a Fourier coefficient, or the velocity at the crest, etc.) is specified. We shall proceed here on the assumption that this is true for three-dimensional waves, and that given $(p, q)$, the mean level, and the waveheight, the infinite system of equations has isolated solutions for the surface shape and the wave speed.

It should be noted that the representation (5) for the velocity potential may not be valid over the entire surface as the singularities in the analytic continuation of $\phi$ into $z>\eta(x, y, t)$ may fall below the crests, in which case the expansion will diverge on those parts of the surface above the singularities (see e.g. Saffman \& Yuen 1982). We shall assume in the present calculation, checked a posteriori by the apparent convergence of the velocity potential at the crest, that this does not occur for the waveheights of our calculations. Otherwise it would be necessary to use some other representation of the velocity potential such as a vortex-sheet formulation. SY approximated the infinite set of equations for the Fourier coefficients by a simpler set due to Zakharov (1968), and demonstrated analytically that the approximated plane Stokes-wave solution bifurcates at critical values of the waveheight, which depend on $p$ and $q$, into non-uniform three-dimensional solutions, examples of which were exhibited. We now describe how the numerical calculation is performed for the full unapproximated water-wave equations.

The calculation is restricted to rational values of $p$ so that the surface has a definite period in the $x$-direction. Collocation is used to generate a truncated version of the infinite nonlinear system. For reasons to be discussed in $\S 3$, the most important case is $p=\frac{1}{2}$, and our attention here is confined to this value of $p$. Fortunately, it is also the easiest to calculate. We alter the labelling of the suffices, scale the $y$-variable, and truncate the expansions for $\eta$ and $\phi$ to give

$$
\eta\left(x^{\prime}, y^{\prime}\right)=\sum_{m=0}^{M} \sum_{n=0}^{N} A_{m, n} \cos \frac{1}{2} m x^{\prime} \cos n y^{\prime},
$$




$$
\phi\left(x^{\prime}, y^{\prime}, z\right)=\sum_{m=1}^{M-1} \sum_{n=0}^{N} B_{m, n} \sin \frac{1}{2} m x^{\prime} \cos n y^{\prime} \exp \left(\frac{1}{4} m^{2}+n^{2} q^{2}\right)^{\frac{1}{2}} z,
$$

where

$$
x^{\prime}=x-c t, \quad y^{\prime}=q y .
$$

In this representation, the regular Stokes wave has wavelength $2 \pi$ and Fourier coefficients satisfying

$$
A_{m, n}=B_{m, n}=0 \quad \text { if } \quad n \neq 0, \quad A_{m, 0}=B_{m, 0}=0 \quad \text { if } m \text { odd } .
$$

Subharmonic three-dimensional bifurcation occurs when solutions exist with $A_{m, n} \neq 0$ for $n \neq 0$ and $m$ odd.

Owing to the even symmetry of (7) it is sufficient that (2) and (3) be satisfied at the discrete set of points

$$
x_{i}^{\prime}=\frac{2 \pi i}{M} \quad(i=0,1, \ldots, M), \quad y_{j}^{\prime}=\frac{\pi j}{N} \quad(j=0,1, \ldots, N) .
$$

In order to fix the wave speed $c$ and Bernoulli constant $K$, we specify a waveheight and mean level through the conditions

$$
\begin{gathered}
\eta(0,0)-\eta(\pi, 0)=h, \\
A_{\mathbf{0}, 0}=0 .
\end{gathered}
$$

Note that $h$ is not in general the waveheight defined as the vertical distance between highest and lowest points, since $\eta(\pi, 0)$ is not necessarily a minimum for the bifurcated waves in which the trough may be displaced relative to the crest. The magnitude of waves is also commonly expressed in terms of the steepness $k a$, where $k$ is the wavenumber and $a$ the wave amplitude. Here, where the Stokes wave has wavelength $2 \pi$, we shall define the steepness $k a$ by the relation $k a=\frac{1}{2} h$. Results will be expressed in terms of either $h$ or $k a$.

The number of unknowns $A_{m, n}, B_{m, n}, c, K$ is

$$
(M+1)(N+1)+(M-1)(N+1)+1+1=2 M(N+1)+2 .
$$

The kinematic equation (2) is automatically satisfied at $x=0$ and $x=2 \pi$ from the symmetry of (7) and (8). Satisfying the kinematic equation at the remaining points gives $(M-1)(N+1)$ equations. The dynamic equation (3) gives $(M+1)(N+1)$ equations from all the points in (10). It is clear that $B_{0, n}$ and $B_{M, n}$ make no contribution to the boundary condition at the points (10), which is why they have been omitted from (8). With the two equations (11) and (12), we then have the same number of equations as unknowns.

Although it has not been proved mathematically that the system of equations is independent, we expect the discrete system to possess isolated solutions in general. This is confirmed numerically by the non-vanishing of the Jacobian except at the bifurcation points.

The $2 M(N+1)+2$ equations were solved by Newton's method, with the wave height $h$ and transverse wavenumber $q$ regarded as parameters. For a specified value of $q$, continuation in the waveheight was performed in order to generate the two-dimensional branch. Bifurcation was detected by monitoring the sign of the determinant of the Jacobian matrix. Values of the critical waveheight for a given $q$ were then refined to four significant figures using bisection. The right and left null vectors at the bifurcation points were calculated using inverse iteration, and the approximate tangent vector of the bifurcated branch was computed using the first of the three methods outlined by Keller (1977). 
For small waveheights, the leading three-dimensional components are $A_{1,1}$ and $A_{3,1}$, representing three-dimensional modulations having $x$-directional wavelength of $4 \pi$ and $\frac{4}{3} \pi$ respectively. It turns out that $A_{3,1}$ is considerably larger than $A_{1,1}$ (typically by a factor of 10), and is therefore used as a measure of the threedimensionality of the wave.

For values of $q$ such that bifurcation occurred at higher values of the waveheight we encountered some difficulty in obtaining an accurate representation of the tangent owing to inherent conditioning problems of the modal approach caused by the exponential dependence on $z$ in the velocity potential, which for large $m$ and $n$ causes large fluctuations in the magnitude of the elements of the Jacobian matrix. In these cases, we constrained the iteration process to generate solutions on the bifurcated branch by initially setting $A_{3,1}$ to an acceptably small value at the bifurcation point and continuing in $A_{3,1}$.

For small values of the waveheight $(h<0.38)$, only a moderate number of modes were needed in order to obtain values of the surface elevation and potential accurate to four significant figures. Typically, we set $N=13, M=5$ at these amplitudes, and the results were obtained on an IBM 3032 computer using double-precision arithmetic. For larger waveheights we set $N=25, M=7$, and these calculations were performed on a CRAY 1 computer in single-precision (64 bit) arithmetic. The effects of modal truncation were monitored by varying $N$ and $M$ and comparing the results. For the steepest waves reported here $(h=0 \cdot 615)$ the results for the height of the interface, the velocity potential on the interface, the phase speed and the Bernoulli constant are accurate to four significant figures. Also, the velocity potential at the crests was examined to check on the convergence of the expansion, and there were no signs of any difficulties.

\section{Results}

$\mathrm{Su}(1982)$ generates in his experiments two-dimensional waves, which if sufficiently steep develop a distinct regular three-dimensional structure that persists for about 10 wave periods. For $90 \%$ of the time, Su observed a configuration denoted as $L_{2}$ in which the modulational wavelength in the direction of propagation is equal to twice that of the two-dimensional wavetrain. This corresponds to three-dimensional waves with $p=\frac{1}{2}$ (period doubling). Su also observed configurations corresponding to $p=\frac{1}{3}$ (period tripling) and $p=\frac{1}{4}$ (period quadrupling) contaminating the $L_{2}$ state for about $10 \%$ of the time. The predominance of the $L_{2}$ configuration can be understood theoretically from the results of MeLean, Ma, Martin, Saffman \& Yuen (1981) (henceforth referred to as MMMSY, see also McLean 1982), who studied the instability of finite-amplitude deep-water waves to fully three-dimensional perturbations using the exact equations. They found that for wave steepness $k a$ greater than $0.30(h>0.60)$ the most unstable disturbance is three-dimensional and occurs for $p=\frac{1}{2}$. It was also noted by MMMSY that these class II (in their notation) disturbances travel with the same speed as the undisturbed wave and that the stability boundaries for $p=\frac{1}{2}$ correspond to stationary disturbances. This property of class II disturbances is also true for $h<0 \cdot 6$, but in this case the most unstable disturbance is of the two-dimensional Benjamin-Feir type (class I). The existence of these class II stationary disturbances implies the occurrence of three-dimensional bifurcations of the two-dimensional Stokes wave. Mathematically, it means that the Frechet derivative is singular at these points; physically, it means that cotravelling disturbances can be superposed on the plane Stokes waves, leading to finite-amplitude three-dimensional waves. 


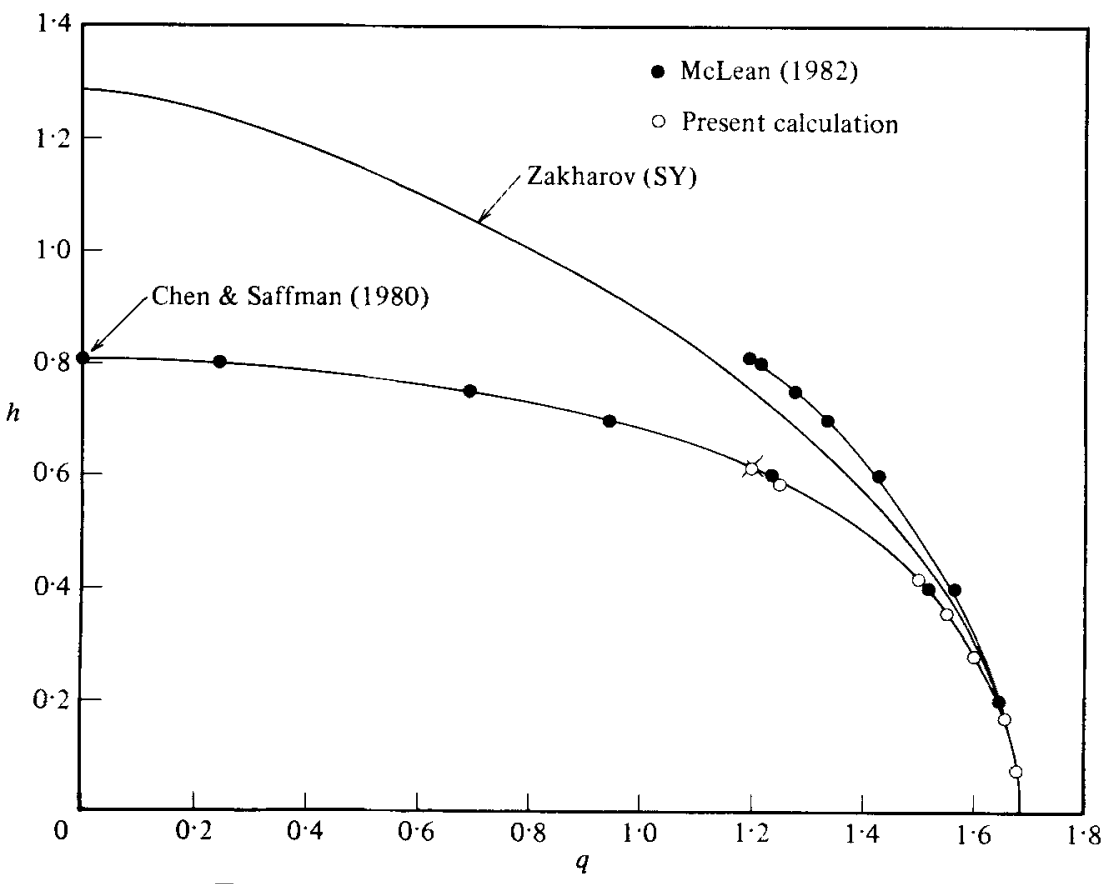

Figure 1. Bifurcation values of $h$ versus $q$ for $p=\epsilon$.

In figure 1 we plot calculated values of the transverse wavenumber $q$ at which bifurcation occurs, found from the vanishing of the Jacobian, as a function of the wave steepness for $p=\frac{1}{2}$. These values tend in the limit of $q \rightarrow 0$ to the critical waveheight $h=0.810$ for the wavelength-doubling bifurcation of two-dimensional waves discovered by Chen \& Saffman (1980), but the present method is not suitable for such steep waves.

We also show in figure 1 the $p=\frac{1}{2}$ stability-exchange values calculated by McLean (1982). The MMMSY instability band is of finite width and there are therefore two values of waveheight (and $q$ ) for a given value of $p$; only the smaller wa veheight values were studied from the standpoint of bifurcation.

In his experiments, $\mathrm{Su}(1982)$ observed wave patterns with $p=\frac{1}{2}$ and $q=1.2$ with a corresponding wave steepness $k a$ of $0.31(h=0.62)$. The bifurcation analysis of the unapproximated equations predicts $h=0.616$ for these values of $p$ and $q$, in excellent agreement with Su's data shown by the $\times$. The fact that Su's value of $h$ is close to that for which the most-unstable disturbance ceases to be two-dimensional (class I) and becomes fully three-dimensional (class II) with $p=\frac{1}{2}$ suggests strongly that the three-dimensional instability leads, when dominant, to a transition to the family of steady three-dimensional waves. Support for this idea would result from a demonstration that the three-dimensional waves are stable to the same class of disturbances, but this is a considerably more extensive calculation and will be left for later study. The actual detailed mechanism of transition from steady two-dimensional to steady three-dimensional waves is outside the scope of an inviscid time-independent calculation, and only qualitative arguments based on stability and energy considerations can be made at this stage.

The degree of three-dimensionality as one follows a bifurcated branch of solutions is measured by the parameter

$$
\epsilon=\frac{A_{3,1}}{A_{\mathrm{c}}}
$$




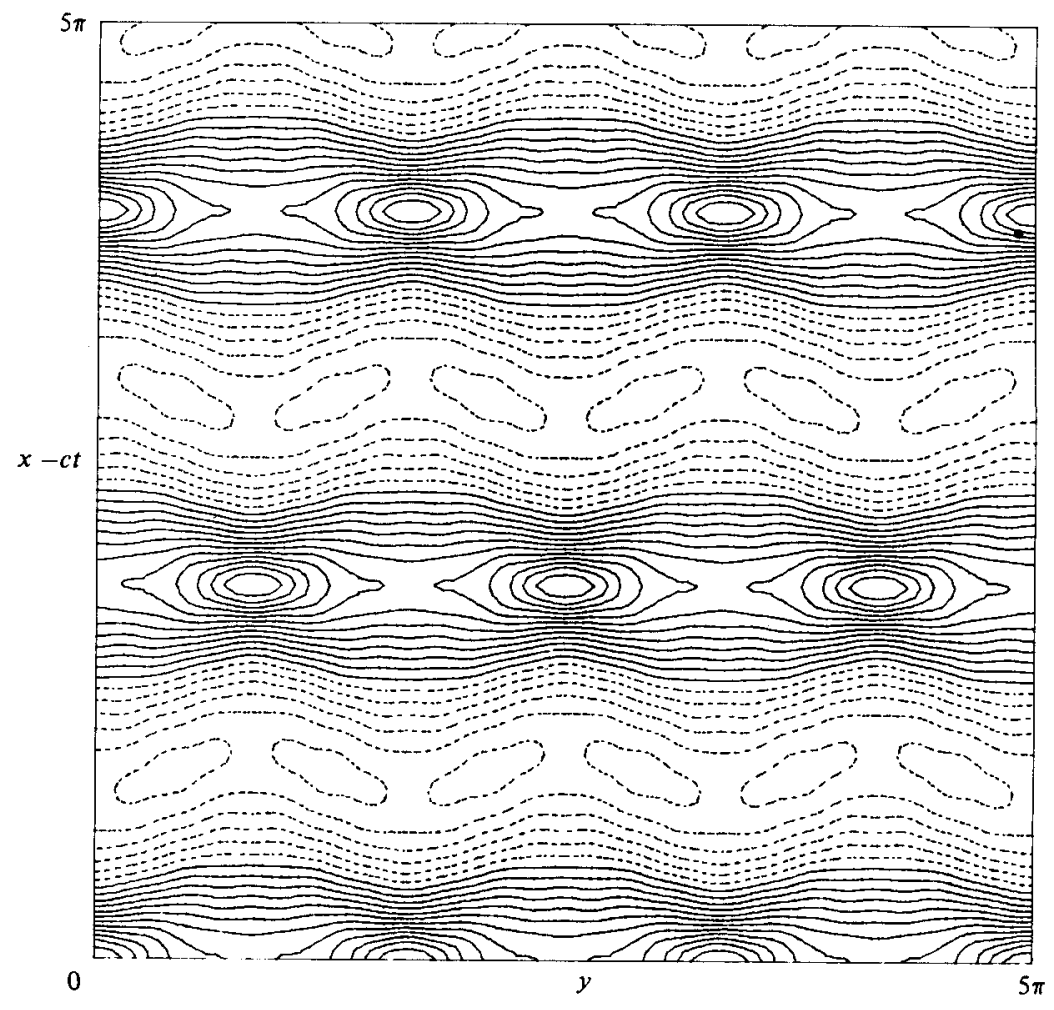

Figure 2. Contour plot for $p=\frac{1}{2}, q=1 \cdot 2, \epsilon=0 \cdot 2$.

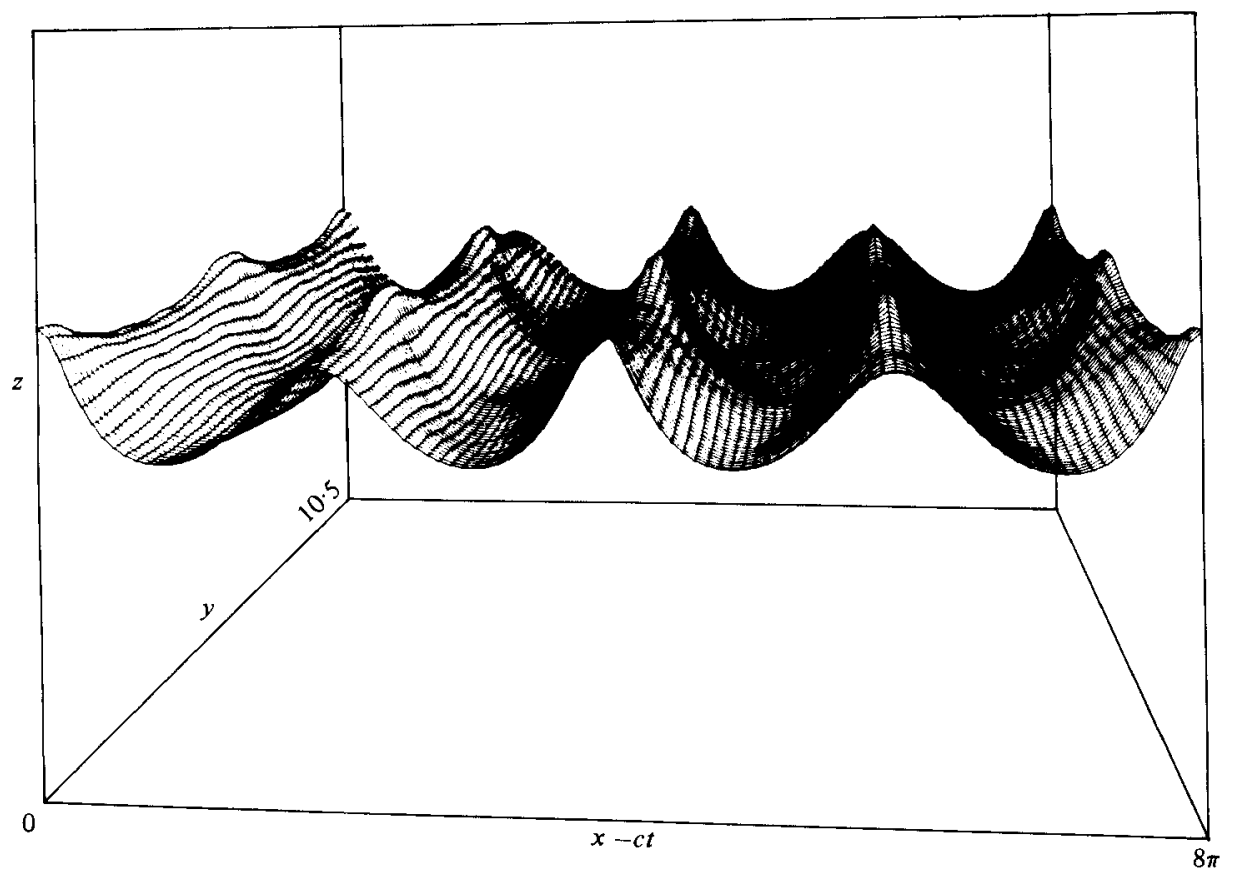

Figure 3. Perspective plot for $p=\frac{1}{2}, q=1 \cdot 2, \epsilon=0 \cdot 2$. 
where $A_{\mathrm{c}}$ is the critical waveheight at which the bifurcation occurs. A contour plot and a three-dimensional perspective plot of the water surface obtained from the unapproximated equations for $\epsilon=0 \cdot 2$ are displayed in figures 2 and 3 respectively. These are to be compared with Su's figures and photographs. The profile traces exhibited by $\mathrm{Su}$ correspond to traverses of our figures 2 and 3 at constant values of $y$. The example discussed in detail by Su corresponds to a value of $\varepsilon=0 \cdot 3$. In our calculations, with 25 modes in the $x$-direction and 7 modes in the $y$-direction, the largest value of $\epsilon$ for which acceptable accuracy could be obtained for $q=1 \cdot 2$ was $\epsilon=0 \cdot 22$. Nevertheless, the qualitative features including the appearance of crescentshaped distortions near the crests of the waves are clearly visible.

In figures 4 and 5 we show plots of the average energy $E$ (sum of kinetic energy in bottom-fixed coordinates and potential energy relative to the mean level), and the height $h$ versus $\epsilon$ for two values of $q$, which determines the critical amplitude at which three-dimensional bifurcation becomes possible. The ratio of $E$ to the average horizontal component of the wave momentum $P$ is almost independent of $\epsilon$ for the values we calculated.

It is interesting to note that for all cases calculated (with $q$ in the range $1 \cdot 2-1 \cdot 6$ ) the momentum and energy of the three-dimensional waves decreases as $\epsilon$ increases (i.e. as the wave becomes more three-dimensional). This suggests that these bifurcated states are favoured by the processes of dissipation, and that in a real fluid, where some loss of momentum and energy must occur, the three-dimensional components will grow together with the waveheight. This could continue until the wave breaks, or it is possible that the energy and momentum could reach a minimum. In the latter case, the real wave would either become irregular in some way or 'snap through' back into a two-dimensional form. Unfortunately, our method of calculation is inadequate to determine whether or not such a minimum exists.

\section{Comparison with weakly nonlinear theory}

In this section we compare the results of the exact equations with those obtained from the approximated system used by SY. For symmetric wave patterns these approximate equations for $A_{l, m, n}$ take the form

$$
\begin{aligned}
& A_{l, m, n}=2^{-\frac{1}{2}}\left[(1+m p)^{2}+n^{2} q^{2}\right]^{\frac{1}{8}} a_{\mathbf{m}} \quad(l=1), \\
& A_{l, m, n}=0 \quad(l>1),
\end{aligned}
$$

where $\mathbf{m}=(m, n)$ and $a_{\mathbf{m}}$ satisfies the equation

$$
\left\{\left[(1+m p)^{2}+n^{2} q^{2}\right]^{\frac{1}{4}}-(1+m p) c\right\} a_{\mathbf{m}}+\sum_{\mathbf{m}+\mathbf{i}=\mathbf{j}+\mathbf{k}} T_{\mathbf{m}, \mathbf{i}, \mathbf{j}, \mathbf{k}} a_{\mathbf{i}} a_{\mathbf{j}} a_{\mathbf{k}}=0,
$$

where the coupling coefficient $T_{\mathbf{m}, \mathbf{i}, \mathbf{j}, \mathbf{k}}$ is a real function of the vector subscripts and is recorded, for example, in Crawford, Saffman \& Yuen (1980). The system is closed by either specifying the waveheight or taking one of the $a_{m}$ as given. The critical values of $a=a_{0}$ for given $p$ and $q$ at which the Stokes wave $\left(a_{\mathrm{m}}=0\right.$ for $m \neq 0$ ) bifurcates into either symmetrical or skewed waves are the roots of a quadratic equation in $a^{2}$ (SY, equation (26)). The values of $h$ versus $q$ for $p=\frac{1}{2}$ obtained in this way are shown on figure 1 . The agreement as $h \rightarrow 0$ is to be expected, since the Zakharov approximation is asymptotically exact as $h \rightarrow 0$.

Symmetric wave patterns were calculated for $p=\frac{1}{2}$ starting from $k a=0 \cdot 37$ $(h=0.74)$. This is the value of $k a$ predicted by the Zakharov equation for Su's experimentally observed values of $p=\frac{1}{2}$ and $q=1.2$ (as compared with the observed 


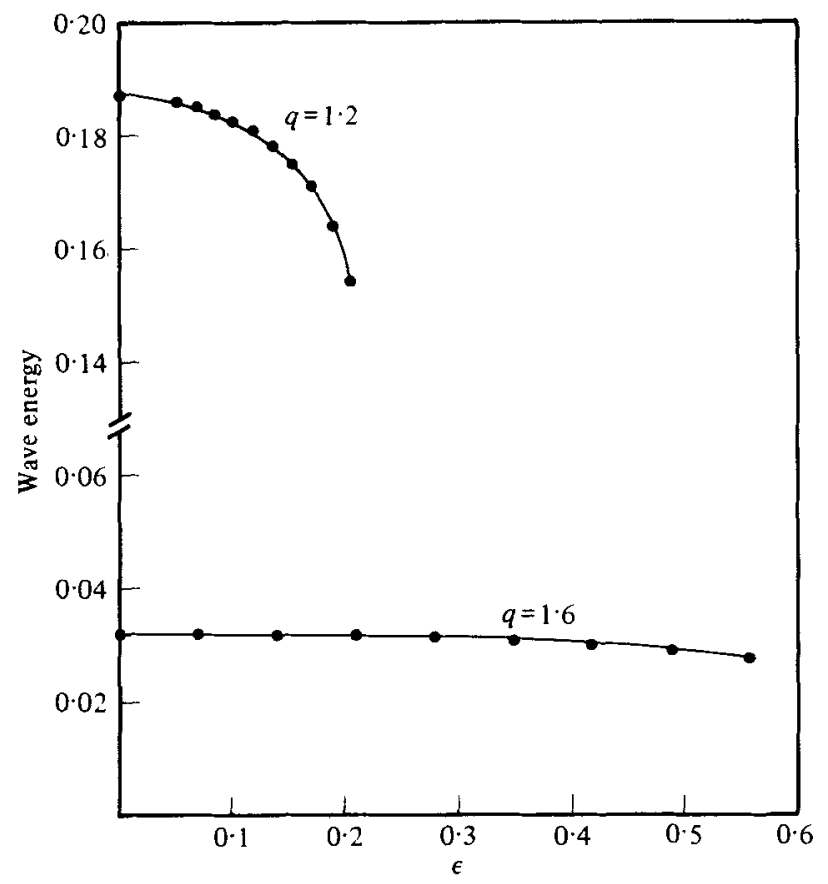

Fratre 4. Average energy $E$ versus $\epsilon$ for $p=\frac{1}{2}$ and $q=1 \cdot 2$ and 1.6.

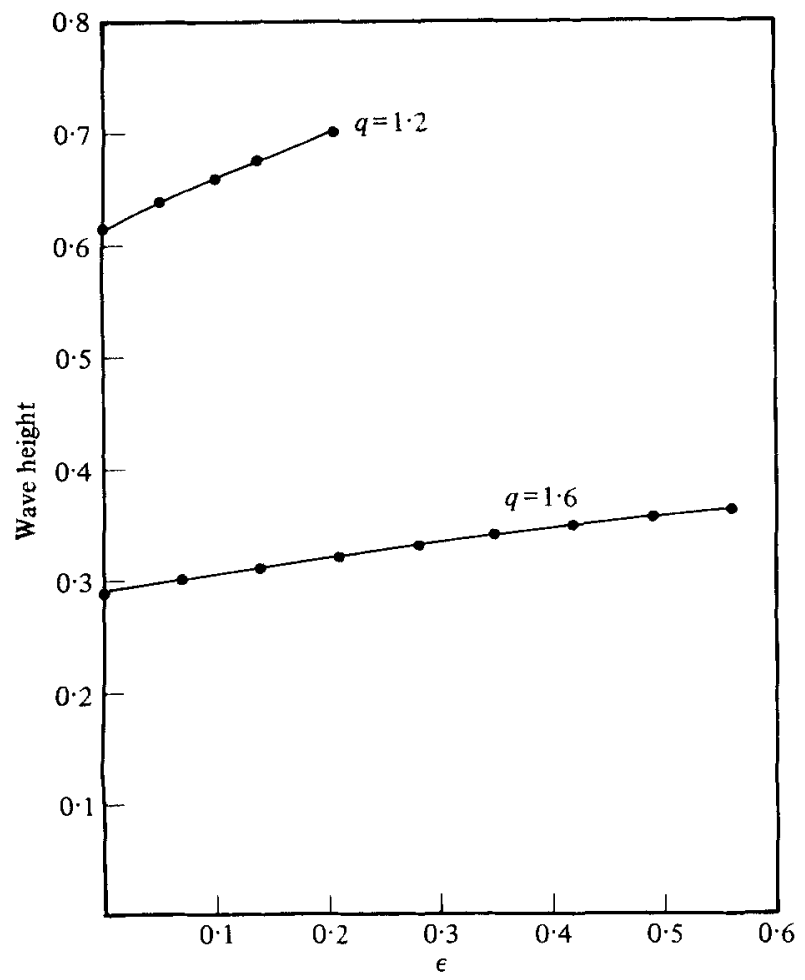

FIGURE 5. Waveheight $h$ versus $\epsilon$ for $p=\frac{1}{2}$ and $q=1 \cdot 2$ and 1.6. 


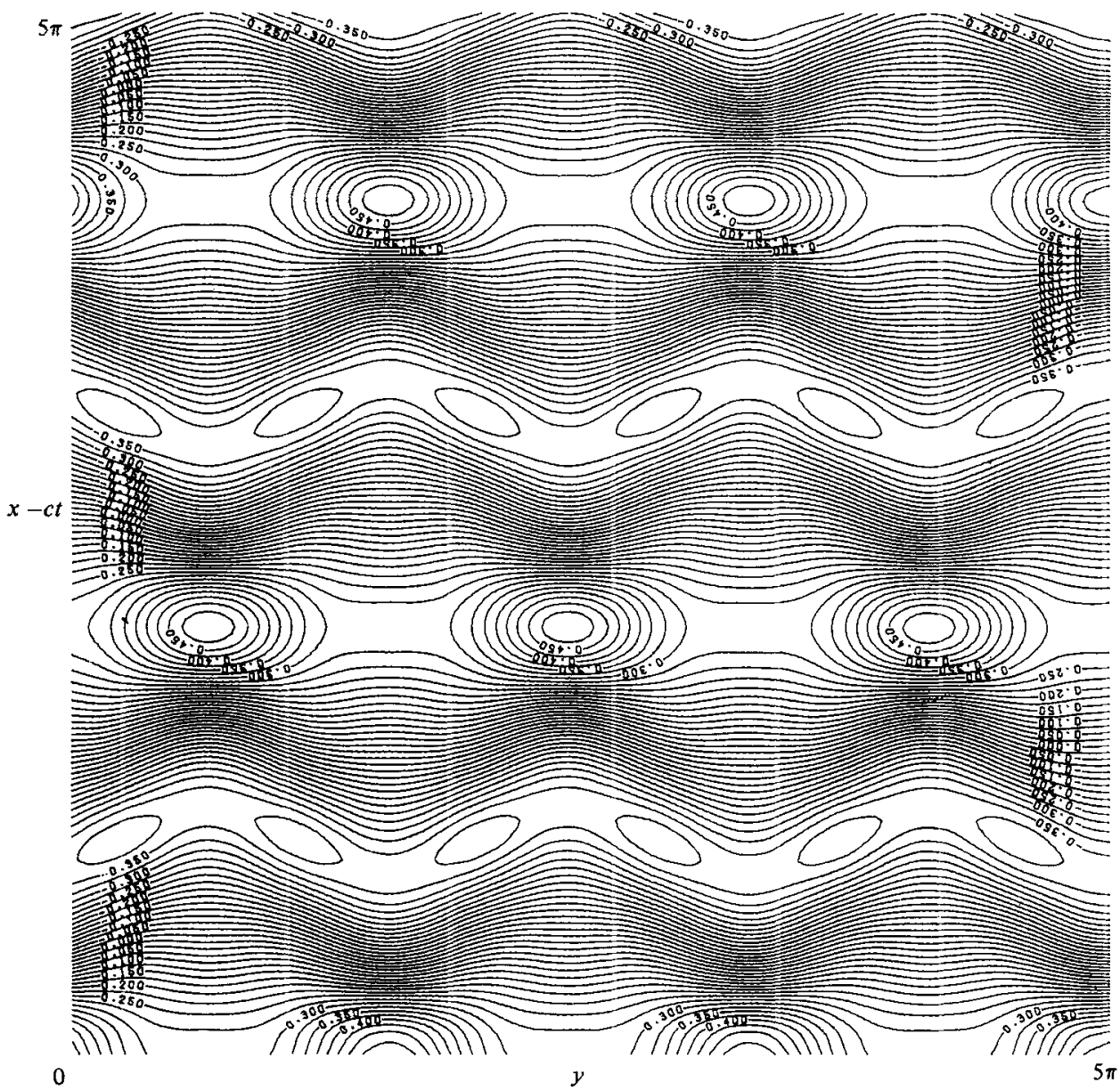

Figure 6. Contour plot of three-dimensional waves given by the Zakharov equation for the same values as above of the parameters.

value of $h=0 \cdot 62$ ). Owing to the cubic nonlinearity of the Zakharov equation, it is possible to solve directly for the modal amplitudes $a_{m}$. The infinite system (17) was truncated to retain modes with $|m|+|n|<N$. The resulting finite system of $(N+1)(N+2)$ equations was solved by Newton's method. The three-dimensional branch is generated by continuing in the parameter $\epsilon$. Calculations were made for values of $N=2,4,6,8$. Accuracy of the results can be estimated by comparing outcomes for different values of $N$. The rate of convergence in $N$ becomes progressively slower as $\epsilon$ increases. However, in view of the approximations already present in the Zakharov equation, we made no serious effort to achieve higher levels of accuracy in its solutions. A contour plot is shown in figure 6 for comparison with the exact solutions shown in figure 2 .

The approximation leading to (17) fails to determine the mode $a_{-P, 0}$ if $p=1 / P$. In this situation, the consistent approach is to set this coefficient to zero and drop the corresponding equation. This procedure was followed here whenever appropriate. 


\section{Bifurcation with $p \neq \frac{1}{2}$}

Su reported the occasional existence of or contamination by three-dimensional structures with triple or quadruple wavelengths, called $L_{3}$ and $L_{4}$ states, corresponding to $p=\frac{1}{3}$ and $\frac{1}{4}$ respectively. In principle, bifurcation can occur for any value of $p$, and can be identified with the stationary states (i.e. disturbances that travel with the same speed as the undisturbed wave) of the MMMSY instability, which were calculated by McLean (1982). Figure 7 shows the plot of $q$ versus $p$ for various values of $h$ at which stationary states occur. Results for $q=0$ given by Saffman (1980) are also included. The predictions of these curves given by the Zakharov approximation are shown in figure 8 for comparison. This approximation is seen to be qualitatively remarkably good.

Owing to the algebraic structure of the Zakharov approximation, calculation of the bifurcated waves is not limited to rational values of $p$, and is easily done for any value. We wish to repeat, however, that there are good physical reasons why only $p=\frac{1}{2}$ should occur, namely that only for this value is the state with stationary disturbances also a state of stability exchange. For $p \neq \frac{1}{2}$ all stability-exchange disturbances propagate with respect to the wave.

The fact that the $L_{3}$ and $L_{4}$ configurations occur rarely and not in pure form, and that they are not favoured by the MMMSY instability mechanism, suggests that these are secondary bifurcations. Indeed, the measured values of $q$ and $k a$ for the $L_{3}$ and $L_{4}$ configurations are not at all consistent with the theoretical results shown in figure 7 .

\section{Do isolated solutions exist?}

We have presented evidence for the theoretical existence of three-dimensional deep water waves of permanent form obtained from the bifurcation of regular Stokes waves and calculated examples. The properties of these waves appear to match those that have been observed experimentally, and there is therefore reason to be confident about the theory and the numerical procedures for amplitudes that are not too large.

However, we wish to point out that despite this success there are some serious theoretical difficulties posing deep mathematical questions which should be raised. It was stated in $\S 2$ that we would proceed on the assumption that the solutions of given wavelength and waveheight are in general isolated, that is, there is no other arbitrarily close solution with the same waveheight and spatial period except at a bifurcation point. For two-dimensional progressive waves there is little doubt that this is true, and indeed it can be proved for sufficiently small waves. However, for three-dimensional waves (cross-waves) this is not so, and power-series expansions in the waveheight run into the small-divisor or resonance problems (see Chappelear 1961). Two-dimensional standing waves are a special case of cross-waves for which the difficulty is easily seen.

Let us consider the so-called primary bifurcation from a flat surface. For progressive two-dimensional waves

$$
\eta=\alpha \cos \left(k x-k^{1} t\right)
$$

is the unique eigenvector of wavelength $2 \pi / k$. (For convenience, we take $g=1$.) The null space is one-dimensional, and for given spatial period there is a family of isolated solutions bifurcating from the flat surface. However, for two-dimensional standing waves

$$
\eta=\sum_{n} \alpha_{n} \cos n^{2} k x \cos n k^{\frac{1}{2} t}
$$



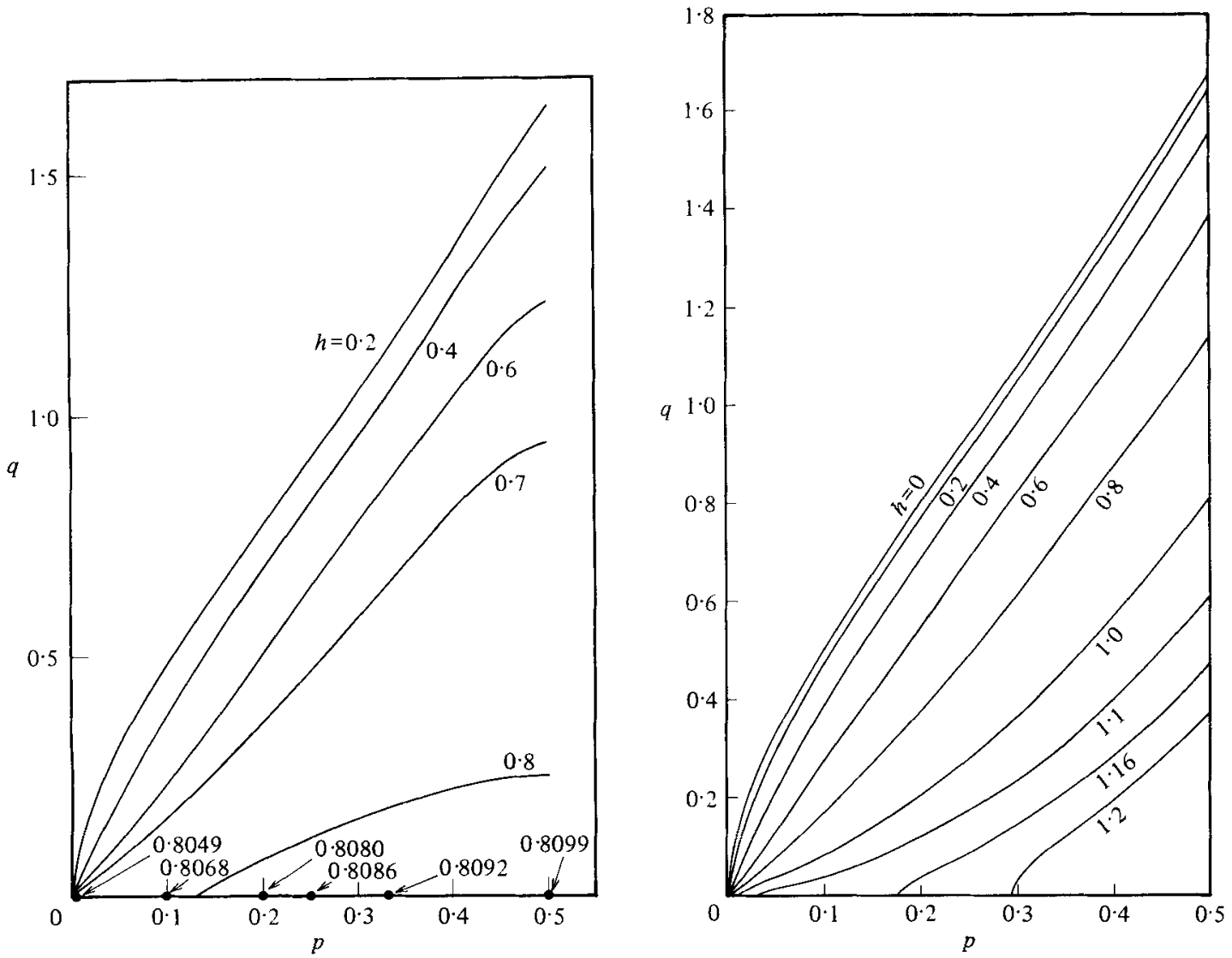

Figure 7. Wavenumber bifurcation loci for various values of waveheight. Numbers with dots on $p$-axis give $h$-values for two-dimensional bifurcation calculated by Saffman (1980).

FIGURE 8. Wavenumber bifurcation loci for various values of waveheight given by the Zakharov approximation.

is an eigenvector of wavelength $2 \pi / k$ for any set of the $\alpha_{n}$ that all have the same temporal period $2 \pi / k^{\frac{1}{2}}$. Thus the null space is infinite-dimensional and the smallamplitude solutions are not isolated. Given any temporally and spatially periodic standing wave it is possible to add another arbitrarily small component, which will not change the waveheight or the magnitude of any particular Fourier component, while still leaving a finite-amplitude standing wave. The requirement that secular terms be absent eliminates some of the arbitrariness, but most of it remains. The expansions by Penney \& Price (1952) and more recently by Schwartz \& Whitney (1981) are approximations to standing waves, but they are by no means the only solutions with the given periods, and their significance is uncertain. In the case of finite depth, the standing waves of small wavelength will have periods that are not exact integer fractions of the period of long-wavelength components, but they can be arbitrarily close. The small-divisor problem appears if an attempt is made to calculate solutions by developing expansions in waveheight. The problem of the existence of isolated solutions is now even more difficult to answer.

The analysis of MMMSY for small-amplitude Stokes waves suggests that there are an infinite number of values of $q$ for each $p$ that will give travelling waves moving with the same speed as the undisturbed wave. For class II disturbances with $p=\frac{1}{2}$ 
these values are

$$
q_{m}=\left(m+\frac{1}{2}\right)\left(m^{2}+m-\frac{3}{4}\right)^{\frac{1}{2}}+O\left(h^{2 m+2}\right) .
$$

Thus there are an infinite number of eigenvectors with the same streamwise wavelength whose transverse wavelength is arbitrarily close to being a rational fraction of the primary transverse wavelength $2 \pi / q_{1}$. Thus the bifurcation is infinitely degenerate and we do not expect that the three-dimensional waves of permanent form constitute isolated families.

This raises two immediate questions: (i) Does this matter physically? (ii) Why doesn't this show up in the numerical calculations? Both questions are answered by the fact that from (20) it is only for large integers $P, Q$ and $m$ that

$$
\frac{q_{m}}{q_{1}} \approx \frac{P}{Q}
$$

Thus the arbitrariness is associated with small-scale components, and is therefore lost in the physical dissipation or the numerical noise. It is curious, however, that we are faced with a situation where increasing the accuracy of a calculation may lead to an increase in the number of close solutions and a decrease in the physical precision.

We can of course ask the hypothetical question: given infinite accuracy, which of the infinite number of solutions of given waveheight and spatial period is of most physical interest? Perhaps a criterion of minimum entropy production, corresponding to minimizing the integral of the sum of the squares of the rate-of-strain tensor, would single out such a solution, although in a strictly inviscid model there is no entropy production. These are all difficult questions, which we are unable to answer, but it was felt appropriate to mention their existence as some of the work on cross-waves ignores them completely.

This work was supported partially by the Office of Naval Research and the National Science Foundation (grant OCE-8100517). The CRAY-1 computer at NCAR used for some of the computations is supported by the National Science Foundation.

\section{REFERENCES}

Chappelear, J. C. 1961 On the description of short crested waves. Army Corps of Engrs, Tech. Memo. no. 125.

Chen, B. \& Saffman, P. G. 1980 Numerical evidence for the existence of new types of gravity waves of permanent form on deep water. Stud. Appl. Math. 62, 1-21.

Crawford, D. R., Saffman, P. G. \& Yuen, H. C. 1980 Evolution of a random inhomogeneous field of nonlinear deep-water gravity waves. Wave Motion 2, 1-16.

Keller, H. B. 1977 Numerical solutions of bifurcation and nonlinear eigenvalue problems. In Applications of Bifurcation Theory (ed. P. H. Rabinowitz), pp. 359-384. Academic.

Molean, J. W. 1982 Instabilities of finite-amplitude water waves. J. Fluid Mech. 114, 315-330.

Mclean, J. W., Ma, Y-C., Martin, D. U., Saffman, P. G. \& Yuen, H. C. 1981 Three dimensional instability of finite amplitude gravity waves. Phys. Rev. Lett. 46, 817-820.

Penney, W. G. \& Price, A. J. 1952 Finite periodic stationary gravity waves in a perfect liquid. Phil. Trans. R. Soc. Lond. A 224, 254-284.

SafFMan, P. G. 1980 Long-wavelength bifurcation of gravity waves on deep water. J. Fluid Mech. 101, 567-587.

Saffman, P. G. \& Yuen, H. C. 1980 A new type of three-dimensional deep-water wave of permanent form. J. Fluid Mech. 101, 797-808. 
Saffman, P. G. \& Yuen, H. C. 1982 Finite-amplitude interfacial waves in the presence of a current. J. Fluid Mech. 123, 459-476.

SChWARTZ, L. W. \& WhitNey, A. K. 1981 A semi-analytic solution for nonlinear standing waves in deep water. J. Fluid Mech. 107, 147-171.

St, M.-Y. 1982 Three-dimensional deep-water waves. Part 1. Experimental measurement of skew and symmetric wave patterns. J. Fluid Mech. 124, 73-108.

ZAKHAROV, V. E. 1968 Stability of periodic waves of finite amplitude on the surface of a deep fluid. J. Appl. Mech. Tech. Phys. 2, 190-194. 